\title{
$A b$ initio electron theory for magnetism in Fe: Pressure dependence of spin-wave energies, exchange parameters, and Curie temperature
}

\author{
S. Morán, C. Ederer, and M. Fähnle* \\ Max-Planck-Institut für Metallforschung, Heisenbergstrasse 3, D-70569 Stuttgart, Germany \\ (Received 26 September 2002; published 27 January 2003)
}

\begin{abstract}
The spin-wave spectra for bcc Fe at various lattice constants are calculated by the ab initio density functional electron theory for various lattice constants, and the exchange parameters of a Heisenberg model are determined from the data. The spin-wave energies increase with decreasing lattice constant, and the behavior of the exchange parameters can by no means be described by the Bethe-Slater curve for an effective nearest-neighbor Heisenberg model. From the spin-wave frequencies the pressure dependence of the Curie temperature is derived in mean-field and random-phase approximation, yielding $\partial T_{c}^{M F} / \partial p \approx 1.6 \mathrm{~K} / \mathrm{kbar}$ and $\partial T_{c}^{R P A} / \partial p$ $\approx 1.8 \mathrm{~K} / \mathrm{kbar}$, in contrast to the experimental result $\partial T_{c} / \partial p \approx 0$. Possible reasons for this discrepancy are discussed.
\end{abstract}

DOI: 10.1103/PhysRevB.67.012407

PACS number(s): 75.30.Ds, 75.30.Et, 75.50.Bb

The exchange interactions in $3 d$ transition metals and their compounds are often discussed within the framework of an effective nearest-neighbor Heisenberg model and the Bethe-Slater curve (see, e.g., Ref. 1) which represents a conjecture on the variation of the nearest-neighbor exchange parameter $J(R)$ with the interatomic spacing $R$. According to this curve, $J(R)$ increases with decreasing $R$ for Co and $\mathrm{Ni}$, whereas it decreases for Fe and changes sign from positive to negative for small $R$. As an example for the application of the Bethe-Slater curve, the temperature dependence of the magnetization of an amorphous ferromagnet was explained ${ }^{2}$ by a mean-field approach with spatial fluctuations of $J$ estimated by use of this curve. If this simple nearest-neighbor Bethe-Slater model was correct, then the critical temperature $T_{c}$ of body-centered-cubic Fe as calculated in mean-field theory would be expected to decrease strongly when pressure is exerted on the sample. Experimentally, however, nearly no change of $T_{c}$ was found ${ }^{3}$ at pressures up to the structural transition from body-centered to face-centered.

Indeed, calculations based on the $a b$ initio densityfunctional electron theory have revealed (Table I) that the simple nearest-neighbor Heisenberg model fails badly for Fe. Instead, an effective Heisenberg model with long-range pair interaction parameters $J_{i j}$ is proposed,

$$
H=-\frac{1}{2} \sum_{\substack{i, j \\ i \neq j}} J_{i j} e_{i} \cdot e_{j},
$$

where $e_{i}$ and $e_{j}$ are the unit vectors pointing in directions of the locāl magnetic moments at sites $(i, j)$, respectively. The exchange parameters remain non-negligible over a very long range, and they oscillate in sign (Table I). It is the objective of this paper to calculate, by $a b$ initio density-functional theory, the change of the spin-wave spectrum with decreasing lattice constant. From this information we then obtain related modifications of $J_{i j}$, and predictions of the meanfield theory and random-phase approximation for the pressure dependence of $T_{c}$.

For the determination of the spin-wave spectra we use the method of Grotheer et al., ${ }^{10}$ which is an alternative to the so-called frozen-magnon method introduced by Halilov et $a l .{ }^{11}$ Thereby, a spiral external field is applied, which for a monatomic unit cell has the form

$$
B_{\underline{T}}^{x}+i B_{\underline{T}}^{y}=B \exp (i \underline{q} \cdot \underline{T}),
$$

where $q$ is the wave vector of the spiral and $\underline{T}$ denotes the translation vectors of the atomic lattice. The field is constant within each atomic sphere. The linear response has the form of a spin spiral,

$$
M_{\underline{T}}^{x}+i M_{\underline{T}}^{y}=M(\underline{q}) \exp (i \underline{q} \cdot \underline{T}),
$$

with

$$
M(\underline{q})=\chi^{\perp}(\underline{q}) B=A^{-1}(\underline{q}) B .
$$

Here

$$
A(\underline{q})=\sum_{\tau} A(\underline{\tau}) \exp (i \underline{q} \cdot \underline{\tau})
$$

is the Fourier transform of the transverse coupling constant $A(\underline{\tau})$. This quantity is related to the exchange parameter $J_{i j}$ of the Heisenberg model [Eq. (1)] via

$$
J_{i j}=M_{0}^{2} A\left(\underline{T}_{i}-\underline{T}_{j}\right),
$$

where $M_{0}$ is the atomic magnetic moment in the ferromagnetic ground state. The magnon frequency $\omega(q)$ is given by

$$
\hbar \omega(\underline{q})=\frac{2 \mu_{B} M_{0}}{\chi^{\perp}(\underline{q})},
$$

where $\mu_{B}$ denotes Bohr's magneton. This equation may be derived from the eigenvalue problem [Eq. (2) of Ref. 10] defined by inserting a plane-wave ansatz into the equation of motion for adiabatic spin-waves, thereby using Eq. (4) i.e., $A^{-1}(\underline{q})=\chi^{\perp}(\underline{q})$.

The calculations are performed by ab initio density functional theory in the local-spin-density approximation ${ }^{12}$ in the parametrization of Ref. 13 and by our recently developed version $^{14-16}$ for noncollinear spin systems of the tightbinding linear-muffin-tin-orbital method in the atomic sphere 
TABLE I. Values for the first four exchange parameters (in $\mathrm{meV}), J_{1}=J_{0 j}$ with $j$ denoting one of the nearest neighbors, $J_{2}$ $=J_{0 j}$ with $j$ denoting one of the next-nearest neighbors, etc. . In Ref. 6 two different sets of values are given, one obtained by the Korringa-Kohn-Rostocker method (KKR), and one by the LMTO method. The data of Ref. 8 are taken from their Fig. 1. The present calculations are for a lattice constant at $a=5.43 a_{0}$. The lattice constants used for the other calculations are not given explicitly in the papers, but they are probably close to this value.

\begin{tabular}{lcccccccc}
\hline \hline$J_{j}$ & $\begin{array}{c}\text { present } \\
\text { work }\end{array}$ & 4 & 5 & $\begin{array}{c}6 \\
\text { KKR }\end{array}$ & $\begin{array}{c}6 \\
\text { LMTO }\end{array}$ & 7 & 8 & 9 \\
\hline$J_{1}$ & 26.80 & 38.91 & 32.65 & 53.60 & 33.20 & 25.85 & 25.03 & 40.95 \\
$J_{2}$ & 22.86 & 22.18 & 18.77 & 17.01 & 17.28 & 20.41 & 22.99 & -6.80 \\
$J_{3}$ & -1.08 & -0.41 & -0.81 & -3.53 & 0.13 & -0.95 & 2.17 & \\
$J_{4}$ & -3.94 & -3.40 & -2.72 & -4.49 & -2.86 & -4.08 & -1.77 & \\
\hline \hline
\end{tabular}

approximation (ASA) ${ }^{17}$ We thereby apply the ASA approximation to the structure and also to the spin direction, ${ }^{18}$ i.e., we take into account for the calculation of the exchangecorrelation energy for each atomic sphere around an atom at site $\underline{T}$ only the projections $\underline{e} \underline{T}^{\cdot} \underline{m}_{T}$ of the magnetization density $\underline{m}_{I}$ on the local spin quantization axis described by the unit vector $\underline{e}_{I}$ (Note: the magnetic moment $\underline{M}_{T}$ is just the volume integral over $\underline{m}_{T}$ ). Because the choice of $\underline{e}_{T}$ has an influence on the directions of the induced moments, the directions of the moments and of the spin quantization axes have to be determined self-consistently. We thereby select, after each iteration step of this self-consistency cycle, the spin quantization axes $\underline{e}_{\underline{T}}$ in such a way that they are parallel to $\partial E / \partial \underline{e}_{\underline{T}}$ evaluated according to Ref. 15. It has been shown ${ }^{16}$ that by this choice the error in the total energy originating from the ASA approximation is minimized. When choosing the spin quantization axes in the conventional way ${ }^{18}$ parallel to the moments after each iteration step, very large errors for $\chi^{\perp}(q)$ (factors 2-3) may arise. To handle the spiral spin configuration we use Sandratskii's extended Bloch theorem. ${ }^{19}$ The results for $\hbar \omega(\underline{q})$ have to be converged very carefully with respect to the magnitude $B$ of the applied transverse field and with respect to the parameters of the Brillouin zone integration. The value of $B$ has to be large enough to exclude numerical uncertainties but sufficiently small to guarantee a linear response of the spin system. It turns out that this is achieved for $B$ values which lead to a canting of the induced spin spiral described by azimuthal angles $10^{-8} \mathrm{rad} \leqslant \vartheta \leqslant 10^{-3} \mathrm{rad}$. Concerning the Brillouin zone sampling it turns out that a $k$-point grid with $N \times N$ $\times N$ lattice points, $N=40$, in combination with a Gaussian smearing procedure ${ }^{20-22}$ with a smearing parameter $\sigma$ with $\sigma N=160$ mRyd is sufficient.

The spin-wave spectra are calculated for the lattice constants $a=5.53 a_{0}, \quad 5.48 a_{0}, \quad 5.455 a_{0}, \quad 5.43 a_{0}, \quad 5.405 a_{0}$, $5.38 a_{0}$, and $5.33 a_{0}$ where $a_{0}$ is Bohr's radius. The results for the spin-wave spectra along the high-symmetry lines in the Brillouin zone are shown in Fig. 1 for a reduced lattice constant $\left(a=5.53 a_{0}\right)$ and for an expanded lattice constant ( $a$ $\left.=5.33 a_{0}\right)$. The experimental lattice constant at room temperature is $5.416 a_{0}$. From the compressibility of $\mathrm{Fe}$ we esti-

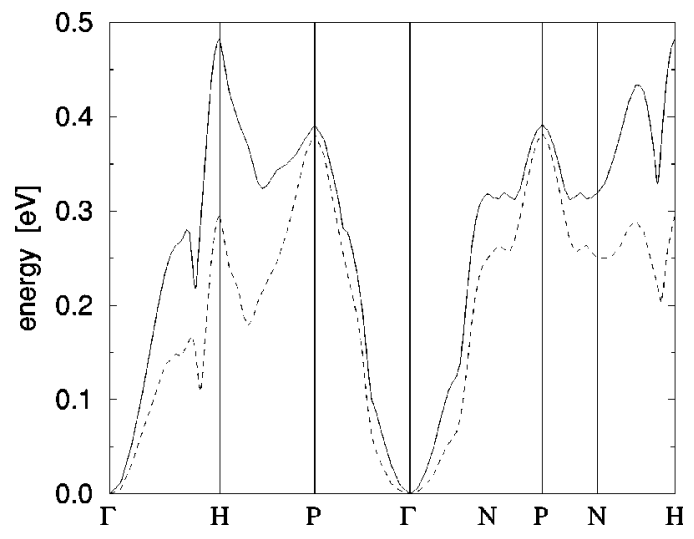

FIG. 1. The calculated spin-wave spectra along high-symmetry directions of the Brillouin zone for bcc Fe at the lattice constants $a=5.33 a_{0}$ (full line) and $a=5.53 a_{0}$ (dashed line). The experimental lattice constant at room temperature is $a=5.416 a_{0}$.

mate a pressure of $83 \mathrm{kbar}$ which would be required to obtain $a=5.33 a_{0}$, which is experimentally accessible. Figure 1 shows that the spin-wave energies increase when the lattice constant decreases. The Kohn anomalies especially around the $H$ point are clearly visible for all considered lattice constants.

For the cubic symmetry of $\mathrm{Fe}$ the spin-wave energies around the $\Gamma$ point may be written as

$$
\hbar \omega(\underline{q})=\left.D \underline{\mid q}\right|^{2}+E^{\prime}|\underline{q}|^{4}+E^{\prime \prime}\left(q_{x}^{2} q_{y}^{2}+q_{y}^{2} q_{z}^{2}+q_{x}^{2} q_{z}^{2}\right)+\cdots
$$

Representing $\hbar \omega(q) /|q|^{2}$ as a function of $|q|^{2}$ and approaching the $\Gamma$ point from various directions yiēlds straight lines. The intersection of these lines with the ordinate axis yields the spin-wave stiffness $D$, and the slopes are given by $E^{\prime}$ when approaching along $\langle 100\rangle, E^{\prime}+\frac{1}{4} E^{\prime \prime}$ when approaching along $\langle 110\rangle$, and $E^{\prime}+\frac{1}{3} E^{\prime \prime}$ when approaching along $\langle 111\rangle$. Table II represents the fitted data for $D, E^{\prime}$, and $E^{\prime \prime}$ as functions of the lattice constant $a$. The spin-wave stiffness $D$ increases with decreasing $a$, and there is an indication that the influence of the quartic terms becomes smaller at the same time.

We determine real-space couplings $A(\tau)$ by inverting Eq. (5). In the following we just represent the exchange parameters related to the real-space couplings by Eq. (6) for the four closest near-neighbor pairs, because they are definitely numerically stable with respect to the details of the inversion. Whereas our method thus is able to determine explicitly only a limited number of real-space couplings, all couplings are accounted for in the calculation of the spin-wave energies

TABLE II. The spin-wave stiffness constant (in $m e V \AA^{2}$ ) and the coefficients $E^{\prime}$ and $E^{\prime \prime}$ (in $\mathrm{eV} \AA^{4}$ ) of the quartic terms in Eq. (8) for various lattice constants $a$ (in $a_{0}$ ).

\begin{tabular}{lccccccc}
\hline \hline$a$ & 5.53 & 5.48 & 5.455 & 5.43 & 5.405 & 5.38 & 5.33 \\
$D$ & 88 & 145 & 159 & 173 & 202 & 205 & 233 \\
$E^{\prime}$ & 0.3 & 0.2 & 0.2 & 0.2 & 0.1 & 0.2 & 0.1 \\
$E^{\prime \prime}$ & -0.9 & -0.8 & -0.8 & -0.8 & -0.8 & -0.8 & -0.7 \\
\hline \hline
\end{tabular}




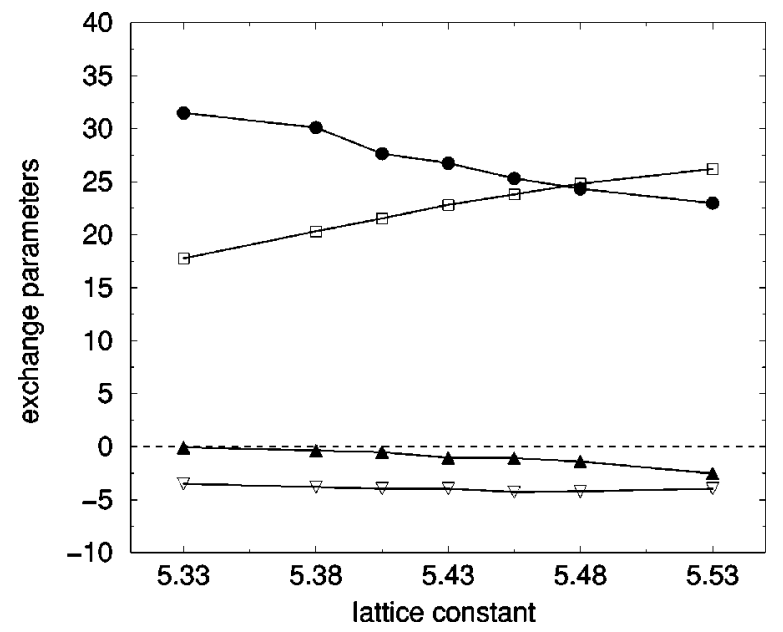

FIG. 2. The exchange parameters $J_{j}$ (in meV) for the four closest near-neighbor pairs as function of the lattice constant $a$ (in $a_{0}$ ). Filled circles: $J_{1}$; unfilled squares: $J_{2}$; filled triangles: $J_{3}$; unfilled triangles: $J_{4}$.

$\hbar \omega(q)$ because periodic boundary conditions based on the exteñded Bloch theorem are used.

Figure 2 shows the exchange parameters for the four closest near-neighbor pairs as a function af $a$. The parameter $J_{1}$ $>0$ for the nearest-neighbor pair is the largest for small $a$, and decreases monotonically with increasing $a$. The nextnearest-neighbor parameter $J_{2}>0$ increases with increasing $a$ and exceeds $J_{1}$ for large $a$, whereas the absolute values of $J_{3}, J_{4}<0$ increase essentially with increasing $a$. Altogether, it is obvious that the situation is much more complicated than the one suggested by the Bethe-Slater curve. It is interesting to compare these results with those given in the paper of Sabiryanov and Jaswal. ${ }^{23}$ In that paper one single atom is displaced toward or away from one of the nearest-neighbor atoms or toward or away from one of the next-nearestneighbor atoms, whereas the position of all the other atoms are fixed. In that situation (which is of course in detail different from our situation) both $J_{1}$ and $J_{2}$ decrease monotonically with increasing interatomic distance.

In the following we calculate the Curie temperature $T_{c}$ by use of the Heisenberg Hamiltonian [Eq. (1)]. The Heisenberg model accounts only for the transverse thermal spin fluctuations which are described by fluctuations of $\underline{e}_{i}$ but not for longitudinal spin fluctuations, i.e., fluctuations in the magnitudes of the atomic moments. For the calculation of $T_{c}$ we use the mean-field result derived from the Heisenberg model,

$$
k_{B} T_{c}^{M F}=\frac{1}{6} \frac{M_{0}}{\mu_{B}} \frac{1}{N} \sum_{\underline{q}} E(\underline{q}),
$$

and the random-phase approximation ${ }^{24}$

$$
\left(k_{B} T_{c}^{R P A}\right)^{-1}=\frac{6 \mu_{B}}{M_{0}} \frac{1}{N} \sum_{\underline{q}} \frac{1}{E(\underline{q})} .
$$

Here the sums run over the $q$ values on a grid in the first Brillouin zone, $N$ is the number of grid points. Although $T_{c}^{M F}$ is expressed in terms of the spin-wave energies, it is well
TABLE III. The calculated Curie temperatures (in K) for various lattice constants (in $a_{0}$ ). For the RPA we give the data obtained by the cube integration, see text.

\begin{tabular}{lccccccc}
\hline \hline$a$ & 5.53 & 5.48 & 5.455 & 5.43 & 5.405 & 5.38 & 5.33 \\
$T_{c}^{M F}$ & 900 & 967 & 1001 & 1035 & 1066 & 1097 & 1150 \\
$T_{c}^{R P A}$ & 490 & 595 & 635 & 675 & 710 & 735 & 790 \\
\hline \hline
\end{tabular}

known that the mean-field theory is based on the notion of single-spin excitations and thus is strictly valid only for $T$ $\gg T_{c}$. In the random-phase approximation it is assumed that the thermal disordering is achieved by the excitation of independent spin waves. In reality there are also other types of excitation modes, and the critical behavior is determined by a coupling of spin excitations on all length scales. In the following, when estimating the dependence of $T_{c}$ on the lattice constant by the mean-field approximation or by the random-phase approximation for the Heisenberg model, we assume implicitly that the critical temperature and its dependence on the lattice constant are determined by transversal fluctuations of single-spin or spin-wave character.

The numerical calculation of $T_{c}^{R P A}$ requires special care because $1 / \hbar \omega(q)$ diverges for $q$ approaching zero. To handle this problem, $\bar{w}$ e replace the sum over the grid points in a small sphere around $q=0$ by an integration, representing $\hbar \omega(q)$ by the quadratic term in Eq. (8). An influence of the higher terms in Eq. (8) was excluded by converging the results with respect to decreasing size of the sphere. Thereby, the surface of the sphere cuts through the Wigner-Seitz cells around the grid points, and for the summation over the grid points outside the sphere we therefore associate weights to the cut Wigner-Seitz cells according to their volumes inside the first Brillouin zone and outside the sphere. Alternatively, we integrate numerically over a small cube around $q=0$, now also using the quartic terms in Eq. (8). This integration requires much care, but the remaining summation over the grid points is simple because a cut of the cells by the cube can be avoided by choosing the size of the cube appropriately. The results of the two methods agree within the numerical error limit for $T_{c}$ of each method which we estimate to be $\pm 10 \mathrm{~K}$.

The data for $T_{c}^{M F}(a)$ and $T_{c}^{R P A}(a)$ are given in Table III, showing that $T_{c}^{M F}>T_{c}^{R P A}$. Thereby, the value of $T_{c}^{M F}$ interpolated for the experimental lattice constant at room temperature, $a=5.416 a_{0}$, is very close to the experimental $T_{c}$ of $1044 \mathrm{~K}$. Both $T_{c}^{M F}$ and $T_{c}^{R P A}$ increase more or less linearly with decreasing a, with $\partial T_{c}^{M F} / \partial a \approx-1275 \mathrm{~K} / a_{0}$ and $\partial T_{c}^{R P A} / \partial a \approx-1450 \mathrm{~K} / a_{0}$, in contrast to the prediction $\partial T_{c} / \partial a>0$ obtained by use of the Bethe-Slater curve. It should be noted that instead of using Eq. (9) $T_{c}^{M F}$ can also be calculated by the equivalent relation $k_{B} T_{c}^{M F}=\frac{1}{3} \sum_{j \neq 0} J_{0 j}$. Within our present theory it is of course awkward to use this latter relation because we can accurately determinate only a small number of parameters $J_{0 j}$, whereas for the calculation of $\hbar \omega(q)$ entering Eq. (9) all parameters are accounted for (see above). Nevertheless, we used this alternative formulation to figure out which exchange parameters $J_{0 j}$ are respon- 
sible for the increase of $T_{c}$ with decreasing $a$, and it turns out that we have to go far beyond the nearest-neighbor and nextnearest-neighbor parameters to obtain the correct magnitude of $\partial T_{c} / \partial a$.

For the pressure derivative $\partial T_{c} / \partial p=\left(\partial T_{c} / \partial a\right)(\partial a /$ $\partial p)\left.\right|_{a\left(T=T_{c}(p), p\right)}$ we obtain, by extrapolating the isobaric thermal expansion coefficient and the isothermal compressibility required for the calculation of $\partial a / \partial p$ from the available literature data, values of $\partial T_{c}^{M F} / \partial p \approx 1.6 \mathrm{~K} / \mathrm{kbar}$ and $\partial T_{c}^{R P A} / \partial p \approx 1.8 \mathrm{~K} / \mathrm{kbar}$. These values are so large that it should be possible to measure them without any problem; however, the experiment ${ }^{3}$ yielded $\partial T_{c} / \partial p \approx 0$. Therefore, if we assume that the experimental results are correct, we must conclude that the pressure dependence of $T_{c}$ in $\mathrm{Fe}$ cannot be described correctly by the Heisenberg model in the meanfield or random-phase approximation; this means that the pressure dependence of $T_{c}$ in $\mathrm{Fe}$ is not dominated by the behavior of the transverse fluctuations of single-spin or spinwave type. Other types of transverse fluctuations which are neglected in the two approximations, or longitudinal spin fluctuations which are totally neglected in the Heisenberg model, are more relevant. For an ab initio calculation of $T_{c}$,

*Electronic address: faehn@physix.mpi-stuttgart.mpg.de; URL: http://physix.mpi-stuttgart.mpg.de/schuetz/elth/electronth.html

${ }^{1} \mathrm{D}$. Jiles, Introduction to Magnetism and Magnetic Materials (Chapman \& Hall, London, 1998).

${ }^{2}$ K.A. Gallagher, M.A. Willard, V.N. Zabenkin, D.E. Laughlin, and M.E. McHenry, J. Appl. Phys. 85, 5130 (1999).

${ }^{3}$ J.M. Leger, C. Loriers-Susse, and B. Vodar, Phys. Rev. B 6, 4250 (1972).

${ }^{4}$ M. Pajda, J. Kudrnovský, I. Turek, V. Drchal, and P. Bruno, Phys. Rev. B 64, 174402 (2001).

${ }^{5}$ S. Frota-Pessôa, R.B. Muniz, and J. Kudrnovský, Phys. Rev. B 62, 5293 (2000).

${ }^{6}$ V.P. Antropov, B.N. Harmon, and A.N. Smirnov, J. Magn. Magn. Mater. 200, 148 (1999).

${ }^{7}$ M. van Schilfgaarde and V.P. Antropov, J. Appl. Phys. 85, 4827 (1999).

${ }^{8}$ D. Spišák and J. Hafner, J. Magn. Magn. Mater. 168, 257 (1997).

${ }^{9}$ A.J. Liechtenstein, M.I. Katsnelson, V.P. Antropov, and V.A. Gubanov, J. Magn. Magn. Mater. 67, 65 (1987).

${ }^{10}$ O. Grotheer, C. Ederer, and M. Fähnle, Phys. Rev. B 63, 100401 (2001).

${ }^{11}$ S.V. Halilov, A.Y. Perlov, P.M. Oppeneer, and H. Eschrig, Europhys. Lett. 39, 91 (1997).

${ }^{12}$ U. von Barth and C. Hedin, J. Phys. C 5, 1629 (1972). therefore, the $a b$ initio spin fluctuation theory of Uhl and Küble ${ }^{25}$ which encompasses both transverse and longitudinal fluctuations, is more appropriate, and first test calculations indeed yielded ${ }^{26} \partial T_{c} / \partial p<0$ when taking into account longitudinal fluctuations, and $\partial T_{c} / \partial p>0$ when neglecting them. Alternatively, Kakehashi ${ }^{27}$ took both types of fluctuations into account by a variational approach to the degenerate-bands Hubbard model, and obtained for reasonable parameters of the model values for $\partial T_{c} / \partial p$ which are larger in magnitude than our value but with a negative sign, again in disagreement with the experiment. Therefore, at present, no theory seems to be able to reproduce the experimental result $\partial T_{c} / \partial p \approx 0$ for the case of Fe. Finally, it should be mentioned that the local-spin-density approximation used in the present calculations fails to describe some important features of bcc Fe, e.g., it does not yield the correct ferromagnetic ground state and the correct sign of the magnetoelastic coupling coefficient $B_{2} \cdot{ }^{28}$ It could be that the computed pressure dependence of $T_{c}$ is thereby affected.

The authors are indebted to O. Grotheer and J. Kübler for helpful discussions.

${ }^{13}$ V. L. Moruzzi, J. F. Janak, and A. R. Williams, Calculated Electron Properties of Metals (Pergamon Press, Oxford, 1978).

${ }^{14}$ M. Liebs, K. Hummler, and M. Fähnle, Phys. Rev. B 51, 8664 (1995).

${ }^{15}$ O. Grotheer and M. Fähnle, Phys. Rev. B 59, 13965 (1999).

${ }^{16}$ O. Grotheer, C. Ederer, and M. Fähnle, Phys. Rev. B 62, 5601 (2000).

${ }^{17}$ O.K. Andersen and O. Jepsen, Phys. Rev. Lett. 53, 2571 (1984).

${ }^{18}$ J. Kübler, K.-H. Hock, J. Sticht, and A.R. Williams, J. Phys. F: Met. Phys. 18, 469 (1988).

${ }^{19}$ L.M. Sandratskii, Phys. Status Solidi B 136, 167 (1986).

${ }^{20}$ C.-L. Fu and K.-M. Ho, Phys. Rev. B 28, 5480 (1983).

${ }^{21}$ C. Elsässer, M. Fähnle, C.T. Chan, and K.M. Ho, Phys. Rev. B 49, 13975 (1994).

${ }^{22}$ O. Grotheer and M. Fähnle, Phys. Rev. B 58, 13459 (1998).

${ }^{23}$ R.F. Sabiryanov and S.S. Jaswal, Phys. Rev. Lett. 83, 2062 (1999).

${ }^{24}$ C.S. Wang, R.E. Prange, and V. Korenman, Phys. Rev. B 25, 5766 (1982).

${ }^{25}$ M. Uhl and J. Kübler, Phys. Rev. Lett. 77, 334 (1996).

${ }^{26}$ J. Kübler (private communication).

${ }^{27}$ Y. Kakehashi, Phys. Rev. B 38, 6928 (1988).

${ }^{28}$ M. Komelj, M. Fähnle, R.Q. Wu, and G.Y. Guo, Phys. Rev. B 65, 144436 (2002). 